remission and greater reductions in inflammatory biomarkers. Recruitment to HICKORY continues in a randomised, placebo controlled induction cohort and a randomised maintenance phase is ongoing.

\begin{tabular}{|c|c|c|c|c|c|}
\hline \multirow{3}{*}{ Week 14} & \multicolumn{3}{|c|}{$\begin{array}{l}\text { Improvement in ES from BL (\%) } \\
n=57\end{array}$} & \multicolumn{2}{|c|}{$\begin{array}{l}\text { No Improvement from BL (\%) } \\
n=73\end{array}$} \\
\hline & $E S=0$ & $E S=1$ & $E S=2$ & $E S=2$ & $E S=3$ \\
\hline & $\mathrm{n}=10$ & $\mathrm{n}=21$ & $n=26$ & $n=15$ & $\mathrm{n}=58$ \\
\hline $\mathrm{RB}=0$ & 90 & 81 & 62 & 33 & 36 \\
\hline $\mathrm{RB}=1$ & 10 & 19 & 27 & 40 & 41 \\
\hline$R B \geq 2$ & 0 & 0 & 12 & 27 & 22 \\
\hline $\mathrm{SF}=0$ & 30 & 33 & 8 & 7 & 2 \\
\hline$S F=1$ & 60 & 33 & 23 & 27 & 22 \\
\hline$S F \geq 2$ & 10 & 33 & 69 & 67 & 76 \\
\hline
\end{tabular}

\section{PWE-072 PHARMACY TECHNICIAN IN THE IBD TEAM MAINTAINS PATIENT SAFETY WHILST FREEING UP PHARMACISTS AND PHYSICIANS}

Anja St. Clair Jones*. BSUH NHS Trust, Brighton, UK

\subsection{6/gutjnl-2018-BSGAbstracts.204}

Introduction We previously demonstrated that incorporating a pharmacist into the Inflammatory Bowel Disease (IBD) team releases doctors' time and improves the consistency and safety of drug monitoring and counselling. We now take the next logical step, by recruiting a pharmacy technician to do the routine drug monitoring and other duties under the supervision of the specialist pharmacist, thereby freeing up clinicians time. We present the outcome of this 3 month pilot.

\section{Methods}

- Provide a blood monitoring service for immunosuppressant therapies of 524 patients on thiopurines and 419 patients on biologics

- Manage the weekly ordering of infusion medication and manage infusion preparation in the pharmacy-led infusion clinic

- Collate current patient information, IBD scores, faecal calprotectin (FCLP) and blood results for the multidisciplinary virtual biologic and immunosuppressant clinic (VBIC) review

- Manage shared care protocols (SCP)

- Ensure comprehensive patient records, identify funds released

Result

- A total of 260 patients were monitored of which 63 patients (24\%) needed to be contacted to provide the blood test

- 48 referrals (18.4\%) were made to the gastroenterology pharmacist:

- 27 patients (10.3\%) due to drug levels outside therapeutic ranges or antibodies

- 9 patients $(3.5 \%)$ had deranged liver function tests

- 5 patients $(1.9 \%)$ had leucopaenia

- 7 patients $(2.6 \%)$ had either raised FCLP levels or anaemia

- Biological medication for 259 patients (average of 20 patients per weekly clinic) was dispensed, ensuring cold chain procedure and accurate stock control. Using the Aseptic NonTouch Technique (ANTT) infusions were prepared, maximising vial sharing, releases nursing staff to undertake cannulation and pharmacists to monitoring and review patients

- 42 VBIC patients were asked to provide a FCLP sample and bloods 2 weeks prior to VBIC. IBD scores were collected during a phone call

- A total of 17 SCP were sent to patient GP's

- All data was entered on the in house database for easy review by the MDT

Conclusions A competent pharmacy technician can safely take over the majority of the drug monitoring and infusion preparation, previously done by our pharmacist. Released funds of $£ 13 \mathrm{~K}$ (lower staffing cost) and cost savings $£ 36 \mathrm{~K}$ (vial sharing) per year are projected.

This represents an increased cost saving, freeing up nursing time and releasing the pharmacist to deal with identified problems and advanced roles within the team (e.g. outpatient clinics, prescribing, helpline queries, counselling patients, TDM) which in term releases clinicians' time (ECCO 2015 Abstract P306). In addition this audit has identified the on-going need for active monitoring of the medications as $1 / 5$ of patients had abnormal results and $1 / 4$ had to be chased up to undertake monitoring at the appropriate interval.

\section{Liver}

\section{OWE-012 NATIONWIDE POPULATION-BASED EVALUATION OF MORTALITY AND CANCER-RISK IN YOUNG PATIENTS WITH ULCERATIVE COLITIS/PRIMARY SCLEROSING CHOLANGITIS} ${ }^{1,2,3,4}$ Palak Trivedi ${ }^{*},{ }^{4}$ Jemma Mytton, ${ }^{4}$ Felicity Evison, ${ }^{1}$ Sivesh Kathir Kamarajah,
${ }^{3}$ Jessamy Reece, ${ }^{3,4}$ Tariq Iqbal, ${ }^{3}$ Rachel Cooney, ${ }^{2}$ Fiona Thompson, ${ }^{5}$ Martine Walmsley,
${ }^{1,2}$ James Ferguson, ${ }^{1,2}$ Gideon Hirschfield. ${ }^{1}$ University of Birmingham, Birmingham, UK; ${ }^{2}$ Liver
Unit, University Hospitals Birmingham NHS Foundation Trust, Queen Elizabeth Hospital,
Birmingham, UK; ${ }^{3}$ Dept. of Gastroenterology, University Hospitals Birmingham NHS
Foundation Trust, Queen Elizabeth Hospital, Birmingham, UK; ${ }^{4}$ Centre for Rare Diseases,
Institute of Translational Medicine, University of Birmingham, Birmingham, UK; ${ }^{5}$ PSC
Support, UK

\subsection{6/gutjnl-2018-BSGAbstracts.205}

Introduction Advancing age is proposed as a risk factor for mortality in primary sclerosing cholangitis (PSC) (Trivedi*Weismuller* et al. Gastro. 2017). However outcomes against a matched control population need evaluation. Our aim was to provide data-driven prioritisation of unmet need by comparing pts. with ulcerative colitis (UC) and coexisting PSC vs an age-matched cohort with UC alone in a stratified outcomes' analysis.

Method A population-based study was performed via linkage to the national Hospital Episode Statistics registry, which records every adult ( $>18 \mathrm{y}$ of age) hospital attendance, admission or clinic event within England since 2006. Across the entire registry we captured all incident cases of UC alone (group I); and UC with an established diagnosis of PSC, or UC diagnosed with PSC subsequently (group 2). Case finding/definition was as per Jess et al (Gastro 2012), by applying ICD10 codes for UC overall (inclusion K51), UC/PSC more specifically (inclusion K51 + K83.0) and excluding other causes of liver injury (K70-77, K80.3/4, B16-19). Cases were captured till 03/2015; follow-up ending 1y thereafter. Event rates (colectomy, colorectal cancer [CRC], liver 